\title{
The impact of the artificial sweetener, acesulfame-k, consumption during pregnancy on postpartum maternal metabolic health and adipose tissue biology in a mouse model
}

\author{
P.E. Bridge-Comer ${ }^{1}$, A. Spada ${ }^{1}$, J. Morton-Jones ${ }^{1}$, M.H. Vickers ${ }^{1}$ and C.M. Reynolds ${ }^{1,2}$ \\ ${ }^{1}$ Liggins Institute, University of Auckland, Auckland, New Zealand and \\ ${ }^{2}$ School of Public Health, Physiotherapy and Sports Science/Conway InstitutelInstitute of Food and Health, University \\ College Dublin, Belfield, Dublin, Ireland.
}

As rates of obesity, diabetes, and related comorbidities have grown, artificial sweeteners (ASs) have risen in popularity. ASs, while promoted as 'healthier' than natural sweeteners, have also been linked to obesity and metabolic disease ${ }^{(1)}$. Preliminary data from our lab suggests that AS intake during pregnancy impairs glucose homeostasis. Limited information exists on the impact of AS intake during pregnancy on the mother's health postpartum (PP). As gestational diabetes (GDM) can predispose the mother and child to type 2 diabetes mellitus (T2DM) in later life ${ }^{(2)}$, we hypothesised both AS and fructose would impair maternal metabolic health PP following maternal consumption during pregnancy.

Pregnant female C57BL/6 mice received standard chow ad-libitum with either water (CD), fructose (Fr;20\% kcal intake), or AS (AS; $12.5 \mathrm{mM}$ Acesulfame-K) throughout pregnancy and lactation $(\mathrm{n}=8 /$ group). These doses were moderate and represent human daily consumption of $330 \mathrm{ml}$ soda or diet soda. These treatments were maintained until pups were weaned ( 3 weeks PP). After weaning, AS and Fr dams received a CD diet for the remainder of the experiment. Body weight, food and water intakes were measured weekly. Oral glucose tolerance tests (OGTT) were undertaken in late pregnancy (Day 18.5), 8 weeks PP and dams were culled at 9 weeks PP. Adipose tissue was dissected and weighed. Samples were collected in 10\% neutral buffered formalin for histological analysis or snap frozen in liquid nitrogen for molecular analysis. Adipocyte size was determined following haematoxylin and eosin staining using ImageJ software. Markers relating to insulin resistance were examined by RT-PCR and expressed as fold change relative to the control group. Data were analysed by one-way ANOVA and repeated measures as appropriate, with Bonferroni post-hoc test.

Body weight and food intake was consistent across all groups throughout pregnancy and PP. During pregnancy glucose intolerance was heightened in the AS and Fr dams versus CD (Area under the curve, 1385 $\pm 88,1713 \pm 144$ vs $1684 \pm 123 ; \mathrm{P}=0.01, \mathrm{P}=0.005$, respectively). At 9 weeks PP, increased glucose intolerance persisted in both AS and Fr dams (Area under the curve, 1313 \pm 105 , $1382 \pm 94$ vs $1189 \pm 114 ; \mathrm{P}=0.03, \mathrm{P}=0.01$, respectively). Despite this, fasting plasma insulin and leptin in the PP dams were not significantly different. Adipocyte size was significantly increased in both the AS and Fr groups PP $(5965 \pm 1135 \mu \mathrm{m} 2,5379 \pm 860 \mu \mathrm{m} 2 \mathrm{vs} 3882$ $\pm 665 \mu \mathrm{m} 2 ; \mathrm{P}=0.005, \mathrm{P}=0.01$, respectively). Gene expression of $\operatorname{Lepr}$ (Leptin receptor) was decreased in the AS group (1.2 \pm 0.2 vs 0.4 $\pm 0.09 ; \mathrm{P}=0.03$ ) compared to CD and Fasn (Fatty acid synthase) was increased in the AS compared to CD and Fr groups (3.7 \pm 0.9 vs $1.03 \pm 0.2,1.1 \pm 0.2 ; \mathrm{P}=0.02)$.

$\mathrm{AS}$ and Fr intake disrupted maternal glucose homeostasis during pregnancy and PP, even after cessation of AS and Fr diets after birth, this was associated with increased adipocyte size and increased expression of Fasn, a marker known to illicit metabolic dysfunction $^{(3)}$. Therefore, ASs may not represent beneficial substitutes to fructose during pregnancy, with the potential to induce GDM-like symptoms in the mothers and increase the risk of T2DM in later life in mothers. As this is a pre-clinical model, further studies in humans would need to be conducted to ensure findings translate to a human setting.

\section{References}

1. Simon BR, Parlee SD, Learman BS, et al. (2013) J Biol Chem 288(45), 32475-89.

2. Plows JF, Stanley JL, Baker PN, et al. (2018) Int J Mol Sci 10.3390/ijms19113342.

3. Moreno-Indias I \& Tinahones FJ (2015) Journal of Diabetes Research :e970375. 